\title{
EchoGéo
}

ECHOGEO- $53 \mid 2020$

Dénominations plurielles. Quand les noms de lieux se concurrencent

\section{La mémoire de la Grande Guerre à travers les odonymes en France}

\section{Clément Millon}

\section{OpenEdition}

Journals

Édition électronique

URL : https://journals.openedition.org/echogeo/20087

DOI : $10.4000 /$ echogeo.20087

ISSN : 1963-1197

Éditeur

Pôle de recherche pour l'organisation et la diffusion de l'information géographique (CNRS UMR 8586)

Référence électronique

Clément Millon, "La mémoire de la Grande Guerre à travers les odonymes en France », EchoGéo [En ligne], 53 | 2020, mis en ligne le 10 décembre 2020, consulté le 11 août 2021. URL : http:// journals.openedition.org/echogeo/20087 ; DOI : https://doi.org/10.4000/echogeo.20087

Ce document a été généré automatiquement le 11 août 2021.

EchoGéo est mis à disposition selon les termes de la licence Creative Commons Attribution - Pas d'Utilisation Commerciale - Pas de Modification 4.0 International (CC BY-NC-ND) 


\title{
La mémoire de la Grande Guerre à travers les odonymes en France
}

\author{
Clément Millon
}

«Je lance aujourd'hui un appel aux maires de France pour qu'ils fassent vivre, par le nom de nos rues et de nos places, par nos monuments et nos cérémonies, la mémoire de ces hommes qui rendent fiers toute l'Afrique et disent de la France ce qu'elle est profondément: un engagement, un attachement à la liberté et à la grandeur, un esprit de résistance qui unit dans le courage ${ }^{1}$.

1 Tel est le souhait émis, à l'été 2019, par le président de la République, Emmanuel Macron, lors de la commémoration du débarquement en Provence. Faire évoluer la mémoire d'une guerre suppose, au préalable, de se poser la question de savoir quelle place la mémoire de ce conflit occupe. Or, il est surprenant de constater que lors des débats sur la loi de 2012 transformant la commémoration du 11 novembre 1918 en un hommage à tous les morts pour la France ${ }^{2}$, la place du souvenir de l'arrêt des combats n'a pas fait l'objet d'une évaluation très extensive. Le texte est adopté en urgence ${ }^{3}$, dans une situation apparente de consensus de l'opinion. Il est vrai que les Français semblent majoritairement conscients de l'oubli dans lequel tombe la Grande Guerre et paraissent donc en accord avec le projet de loi : 64 \% des Français l'approuvent en novembre 2011, selon l'article précité. En 2012, il s'agit néanmoins, à la veille de l'élection présidentielle, de poser pour l'avenir une réforme qui allait être durable, alors que François Hollande, candidat, y était hostile ${ }^{4}$.

2 Le temps du souvenir est passé. Ceux qui pouvaient témoigner ont disparu, ce qui ne manque pas d'avoir des conséquences pour le couple aux relations compliquées formé par histoire et mémoire (Beaupré, 2014). La commémoration du centenaire de la Grande Guerre a entraîné le développement d'une profusion d'événements mémoriels marquant l'empreinte du mandat de François Hollande, mais qui ne masquent pas que la Grande Guerre appartient désormais, et plus que jamais, au passé de la France. Or, perdre le souvenir de l'histoire de la Grande Guerre, c'est voir s'envoler la mémoire, fragile, du XX $X^{\mathrm{e}}$ siècle dans les jeunes générations (Reynié, 2015). Il est inutile de s'en affoler et vain de le regretter ; il faut d'abord dresser un état des lieux de la persistance de la mémoire du siècle passé. 
3 En matière de politique mémorielle, « chaque nation cherche sa voie » (Ferenczi, 2002, p. 14). La France attache traditionnellement beaucoup d'importance à donner à ses voies un nom plein de sens. Cette question, loin d'appartenir au passé, est d'une actualité brûlante. En effet, les fusions et rassemblements de communes provoquent des doublons que les municipalités doivent éliminer en débaptisant certaines dénominations pour en choisir d'autres. Les édiles peuvent continuer à rappeler les conflits passés à travers les dénominations de voies. Le président de la République peut encourager à souligner l'importance d'un combattant, pour son origine. Mais pour que le nom de rue s'installe dans la mémoire, encore faut-il que la personne soit identifiable par tous. En matière de travail de mémoire, «Il faut bien se rendre compte que lorsqu'on entend ces appels contre l'oubli ou en faveur du devoir de mémoire, ce n'est, la plupart du temps, pas à un travail de recouvrement de la mémoire, d'établissement et d'interprétation des faits du passé qu'on nous invite (rien ni personne, dans des pays démocratiques comme les Etats d'Europe de l'Ouest n'empêche quiconque de poursuivre ce travail), mais plutôt à la défense d'une sélection de faits parmi d'autres, celle qui assure à ses protagonistes de se maintenir dans le rôle de héros, de victime ou de moralisateur » (Todorov, 2001, p. 7). De fait, cela prolonge le conflit qui est pourtant achevé et n'empêche pas la mémoire de s'effacer progressivement.

En France, beaucoup de voies offrent les traces d'une mémoire des guerres, soit par mention d'un conflit, soit par celle d'un de ses acteurs principaux. Il appartient aux conseils municipaux d'adopter une dénomination pour les rues, voies, places, etc., sous le contrôle du préfet. L'étude de cette attribution, odonymie, est d'un grand intérêt national, dès lors que les appellations retenues dépassent, comme en l'espèce, les enjeux locaux. Si l'on a pu faire le constat, à la fin des années 80 , que « entre toutes les disciplines onomastiques, l'odonymie est sans doute la plus délaissée » (Milo, 1989, p. 6), des études se sont penchées, depuis, sur ce champ de recherche assez neuf (Bouvier et Guillon, 2001 ; Badariotti 2002 ; Oulmont 2009 ; Bouvier 2013).

5 La mise à disposition du fichier FANTOIR (2016) a changé la donne, même si, comme nous l'avons fait pour le travailler, il impose des choix de critères de recherche et suppose de s'appuyer sur d'autres sources de façon concomitante. De cette manière, nous avons $\mathrm{pu}$ dresser, département par département, un inventaire des dénominations relatives à la Grande Guerre, dont les résultats figurent en annexe et qui sont commentés ici. De façon plus prosaïque, cette étude est née d'une observation consécutive aux premiers inventaires. Dans le Nord de la France, la commémoration du 11 novembre, événement éphémère, est souvent appuyée par un nom de rue, trace pérenne, qui prolonge la visibilité de l'acte mémoriel. Dans d'autres régions, il faut faire le constat que peu de rues portent la mémoire de la Grande Guerre. Par ailleurs, si la mémoire de la Grande Guerre, par l'attribution de noms de rues, places, boulevards ${ }^{5}$, est très présente après le premier conflit mondial, on peut se demander ce qu'elle représente aujourd'hui.

Poser la question de la place de la Grande Guerre est essentiel, car la mémoire de ce conflit est occultée par celle d'autres épisodes sanglants. Cela s'explique et se justifie aisément par la violence et l'ampleur de la seconde guerre mondiale, notamment. Mais que peut-il rester des autres guerres du $\mathrm{XX}^{\mathrm{e}}$ siècle si la mémoire de la matrice de celleci est oubliée? Cette question impose de soulever la question suivante, d'apparence simple, mais aux implications pourtant complexes :

Que reste-t-il de la Grande Guerre dans la mémoire des rues de France? 
$7 \quad$ Notre étude des sources montre que la place de la Grande Guerre est importante, mais surtout marquée par la souffrance et le deuil (première partie). La mémoire de la Grande Guerre est éclipsée par celle, beaucoup plus présente, de la seconde guerre mondiale (deuxième partie). Enfin, les noms retenus par les municipalités montrent que la place de la mémoire des hommes dépasse celle de la guerre (troisième partie).

\section{Les odonymes rappelant le souvenir de la Grande Guerre}

8 La Grande Guerre est présente dans les noms de rues. Beaucoup de dénominations évoquent le 11 novembre (1909). Mais il faut ajouter celles portant le vocable «Armistice» (43), ou encore «Victoire » (407), ce qui porte le total pour la Grande Guerre à 2359 odonymes.

9 La présence de la Grande Guerre est très inégale selon les départements, avec une forte présence là où se sont déroulés les combats et l'occupation allemande : 113 dans le Pasde-Calais; 99 dans le Nord; 70 dans la Somme, 24 dans l'Oise. La pratique des commémorations est d'ailleurs très vivace dans les régions ayant eu le plus à souffrir de la Grande Guerre (Hardier et Rochas, 2016). Ce constat souffre quelques exceptions. Ainsi, l'Indre-et-Loire place 78 fois le 11 novembre dans ses localités. Mais les départements très touchés par le conflit que sont l'Aisne (19), l'Oise (24), la Meuse ( 1 fois!), peu ou très peu. Les départements alsaciens ignorent carrément une date qu'ils ont peu connu, il est vrai: une seule rue est consacrée dans les deux départements au 11 novembre, à Ingwiller.

10 Nos édiles ont fait principalement le choix de rappeler la date d'un conflit, dont il est intéressant de saisir la portée. Le 11 novembre est donc l'odonyme le plus retenu. Cette date marque en 1918 la fin du conflit. Selon sa définition, un armistice est une simple trêve entre deux adversaires et il ne signifie pas la fin des souffrances liées à la guerre. C'est en effet un jour d'allégresse, mais aussi de deuil, particulièrement dans les régions récemment libérées, comme le Nord (Millon 2019). Ainsi, pour la France, il faut garder « à l'esprit le double caractère de cette journée » (Rémond, 2002, p. 50), qui existe dès 1918 et qui perdure. C'est le deuil qui domine dans la pratique mémorielle.

11 Le rappel de l'armistice du 11 novembre sous ses différentes formes ${ }^{6}$ est attaché au souvenir douloureux de la guerre et de ses blessures humaines, à la victoire qui est le résultat annoncé du conflit, mais surtout au culte des morts qui naît autour du 11 novembre. Le 11 novembre 1918 n'est pas toujours célébré en tant que tel, en 1918, mais devient une date symbolique, moment de communion nationale et mémorielle, avec son culte, ses officiants et ses adeptes. Les odonymes rappellent plutôt la date de la célébration de l'armistice que l'armistice lui-même. C'est d'ailleurs ce qui explique la faible présence de l'occurrence "Armistice». L'importance de ce terme est parfois oubliée. René Rémond rappelle pourtant que « c'est la mention Armistice qui figure sur le calendrier des postes" (Rémond, 2002, p.51). Le terme est entaché par son utilisation, en 1940, pour l'acte du 22 juin qui fonde les relations entre l'Allemagne nazie et la France de Vichy et qui est devenu synonyme de capitulation. Il reste une marque de défaite.

12 La «Victoire", elle ${ }^{7}$, n'est guère présente, à côté de la notion consacrée de 11Novembre. Elle reste une expression largement attachée, au xxe siècle, à la France face à 
l'Allemagne. Elle est acquise le 28 juin 1919. Ainsi, l'Aisne, dont 19 localités seulement célèbrent le 11 Novembre, fête la Victoire en donnant 14 fois ce nom à une voie, dont 4 fois, comme pour le 11 Novembre, à une place. C'est souvent une place qui est nommée ainsi, car c'est là que se déroulent les cérémonies commémoratives. C'est d'ailleurs ainsi qu'il faut comprendre la loi du 24 octobre 1922 «fixant la commémoration de la victoire et de la paix $»^{8}$.

C'est ainsi que des vocables anciens prennent un sens nouveau. De même, la paix a un sens ambivalent. La célébrissime rue de la Paix à Paris est ainsi nommée en 1814 afin de saluer la paix retrouvée, alors que cette dénomination remplace la mention "rue Napoléon ». L'odonymie illustre plus qu'elle ne révèle que la France connaît, au-delà de la victoire ou de la défaite, une culture du «deuil mélancolique » (Largeaud, 2006, p. 354). La rue de la Paix qui traverse les premier et deuxième arrondissements, mène alors aux Tuileries. C'est le chemin parcouru par les ambassadeurs, qui pour beaucoup d'entre eux représentent les vainqueurs coalisés contre l'empereur déchu. Il est paradoxal qu'une telle paix soit célébrée au cœur de Paris, sur ce qui est bientôt un symbole de l'aisance et du chic parisien et dont la réputation mondiale est relayée par le non moins connu Monopoly. L'appellation n'a pas changé, ce qui contribue à la renommée du lieu. Une autre paix s'impose en 1919 : le traité de Versailles est fêté de façon grandiose par le défilé de la Victoire du 14 juillet 1919. Sur le passage du cortège, la rue de la Paix est pavoisée de façon somptueuse, afin d'honorer une paix bien plus favorable à la France. À Strasbourg, la rue de la Paix est célèbre pour avoir accueilli en 1919 le maréchal Foch, chef militaire des armées alliées.

14 Avec l'introduction de nombreuses références au premier conflit mondial dans les localités, le village entre souvent dans une nouvelle phase, qui tient compte de l'Histoire. On rompt avec « cette opposition véritablement structurale entre le Haut et le Bas, qui organisait politiquement le village jusqu'à la guerre de 1914 » (Zonabend, 1980, p. 306). Une mémoire nouvelle s'ancre dans le paysage français : la Grande Guerre est signe de recueillement et deuil. C'est ainsi qu'il faut comprendre la domination du 11 Novembre sur les termes de Paix, Victoire et Armistice. De même, la première guerre mondiale est présente à travers les odonymes rappelant les batailles. Mais ces dernières sont symboles de destruction et les victoires qu'elles représentent sont dépassées par le deuil : Verdun, la Marne, les Flandres. Nous avons renoncé à recenser ces odonymes car il était difficile de les rattacher systématiquement aux batailles, plutôt qu'aux lieux concernés.

\section{Le dépassement de la mémoire de la Grande Guerre}

15 La place de la Grande Guerre est aujourd'hui détrônée par celle d'autres conflits, particulièrement celle de la seconde guerre mondiale. D'abord, la signification évidente d'un terme en 1918 peut changer par une précision ajoutée à l'odonyme. C'est ce que prouve l'existence, rare néanmoins, d'une avenue de la victoire du 8 mai 1945 à Grasse, dans les Alpes-Maritimes ou à Toulon, dans le Var. La Grande Guerre devait être la Der des Der. Qui pouvait prévoir qu'il y aurait un 8 mai 1945 ?

Nous avons vu que la paix pouvait être attachée à la fin de la Grande Guerre. Cependant, dans leur immense majorité, les rues de la paix font référence à une paix des armes qui suit un conflit. Lorsque la paix célébrée ne suit pas la Grande Guerre, mais explicitement un autre conflit, cela est précisé dans un souci d'offrir une part de 
mémoire aux habitants concernés. Tel est le sens de "l'avenue de la paix en Algérie " inaugurée dans le Loiret (450089). La paix retrouvée, les nombreux anciens combattants $\mathrm{y}$ sont très attachés. En effet, près de trois millions de jeunes français sont appelés pour les " événements d'Algérie » auxquels on ne voulait pas donner le nom de guerre. Audelà de la question des rapatriés et autres harkis, peu nombreux dans le Loiret, donner un nom de voie à la paix en Algérie permet de ne pas donner l'impression qu'après leur avoir volé leur guerre, les conscrits de 1954-62 ne se voient pas voler leur paix. La paix n'est pas que l'absence de conflit armé, comme en témoigne les deux rues de la paix sociale dans le département des Ardennes. Le département compte en outre deux lieuxdits nommés « L'arbre de la paix » et « la croix de paix ». La paix devient une référence plus large, un principe ${ }^{9}$. La dénomination « rue de la Paix » a ainsi un sens analogue aux odonymes consacrés à l'égalité, ou à la fraternité.

Le 11 Novembre est surtout radicalement supplanté par la référence au 8 mai, sous toutes ses formes, qui compte 3446 occurrences. Pratiquement tous les départements célèbrent cette date par l'adoption dans une localité de cette dénomination, à l'exception de quelques-uns, rétifs semble-t-il à adopter une odonymie mémorielle, tels que les Hautes-Alpes ( 3 et 17), la Corse-du-Sud (0 et 3 ) et la Haute-Corse (0 et 9), le Lot (11 et 40), la Lozère (5 et 12).

, la notion de «Libération » permet à la seconde guerre mondiale de s'imposer dans la mémoire des rues en France. L'année 1918 est synonyme de libération pour de nombreuses localités en France dont le territoire était occupé, sans compter les départements de l'Est, annexés, qui recouvrent la souveraineté française. Pourtant, les mentions des dates de libération sont rares. En revanche, la Libération est souvent mentionnée par l'odonymie (2 090 voies évoquent la libération de 1944 à 1945), en mentionnant parfois une date, au point de dépasser la présence d'une autre date étudiée, celle du 11 novembre, présente 1909 fois. La libération est souvent l'événement majeur des régions concernées par les opérations de guerre. Ainsi, un grand nombre de départements ont plus de 50 odonymes de ce type, comme par exemple la Charente-Maritime, la Gironde, la Seine-et-Marne ou qui ont été marqués par des combats achevés par la libération (Le Var, les Bouches-du-Rhône, le Calvados). La Marne, pourtant marquée par les combats de la première guerre mondiale et lieu de signature de l'armistice de la seconde, a pour odonyme le plus courant, dans notre sélection, la Libération (45 fois).

19 Si l'on sépare le 8 mai (3 446 odonymes) de la libération (2 090 odonymes), nous trouvons un certain équilibre, après la mention de la première date, entre les mémoires des différents aspects du conflit que sont «Libération» et "11 Novembre». En revanche, en additionnant toutes les références, le total penche fermement en faveur du second conflit mondial. En effet, en ajoutant la référence à la résistance (1 189 odonymes), le second conflit mondial regroupe 6725 odonymes contre, au maximum, 4 108, parfois discutables, sur la Grande Guerre, en mêlant 11 Novembre, armistice, Victoire et Paix.

20 La Résistance est typiquement un phénomène attaché au second conflit mondial, même s'il y a eu une résistance lors du premier. L'odonyme associé à la résistance montre la prégnance du second conflit mondial, par rapport au premier. Sans compter le nom des résistants connus (comme Jean Moulin) ou d'autres, moins célèbres, de nombreux odonymes se rapportent à la Résistance, aux résistants et à ses martyrs. Mais il s'agit déjà de la mémoire d'hommes, plus de celle d'un conflit. 


\section{De la mémoire de la guerre au souvenir des hommes}

L'odonymie ne fait pas que célébrer la mémoire de la guerre. Elle porte sur la mémoire des hommes qui l'ont faite. D'ailleurs, les odonymes n'utilisent que très peu le terme de guerre (47 mentions). De plus, c'est souvent pour en mentionner un autre aspect, comme le fait la « rue de la fin de la guerre » à Tourcoing, le « Rond-point de la Croix de Guerre » de Céret ou encore la «rue des victimes de guerre » à Alquines. Ce sont déjà des hommes qui sont honorés, plutôt qu'un conflit armé.

Ainsi, pour la Grande Guerre, les poilus ont été mis à l'honneur, mais de façon peu courante (143 odonymes). Seuls trois départements, le Nord, le Var et les AlpesMaritimes honorent la mémoire des poilus, ce qui est une façon de saluer les anciens combattants. De toutes les façons, pour ce qui est des poilus, ce n'est pas l'odonymie qui est la plus révélatrice de la place de leur mémoire. Les monuments aux morts et autres plaques communales qui sont présents dans quasiment toutes les localités (Prost, 1984, p. 223) se chargent de leur rendre un culte, quand ce n'est pas les églises, universités, établissements avec leurs plaques, monuments et mémoriaux. Il ne faut pas oublier, en effet, que «les municipalités ne sont pas les seules à rendre hommage aux disparus » (Rémond, 2002, p. 51).

C'est la guerre et ses combattants qui font l'objet d'une commémoration. Cette sacralisation d'un phénomène guerrier est étrangère à nombre de Français du début du $\mathrm{XXI}^{\mathrm{e}}$ siècle. Elle ne permet de transmettre des connaissances sur la première guerre mondiale que de façon marginale, soit $7 \%$ des jeunes en 2014 , contre $24 \%$ pour les œuvres de fiction (Reynié 2015, p. 78). À la fin du centenaire de la Grande Guerre, il est opportun de s'interroger sur la place actuelle de la pratique de commémoration de la Grande Guerre. Elle dit ce qu'était ce culte au siècle précédent. Elle permet de comprendre ce qu'il est devenu face aux mentions d'autres conflits, qui ont dépassé cette mémoire pourtant douloureuse. Elle explique enfin, ce qu'il reste de la mémoire d'un jour que les anciens considéraient souvent comme sacré.

4 Les odonymes consacrés aux anciens combattants entrent dans cette logique. On en dénombre 965, dont 695 restent assez généraux pour saluer la mémoire des combattants de différents conflits. Une catégorie à part se dessine, qui salue l'engagement des anciens combattants en Afrique du Nord ou Indochine. Bien rares sont les odonymes tenant compte d'un lieu, comme le «rond-point des anciens combattants de l'Union française ", à Isle, dans la Haute-Vienne. Les combattants sont mentionnés pour la mémoire du lieu de leur engagement et non leur propre origine.

Les références aux «martyrs de la résistance » ne se rencontrent que dans les mairies de gauche à la libération (Panicacci, 2001, p. 97). Ce constat général peut être relativisé pour d'autres villes. Des communes d'autres couleurs ont adopté cette dénomination dans d'autres régions, à tout le moins ${ }^{10}$. Quoiqu'il en soit, cette appellation répond à une motivation largement identifiable pour les édiles de gauche : « ils entretiennent la mémoire de leurs compagnons de lutte en légitimant leur propre pouvoir» (Desbrosse, 2014, p. 114). Il ne s'agit donc pas, ou pas seulement, de faire mémoire d'un conflit.

6 S'il y a une bataille de la mémoire à propos de la Grande Guerre, c'est entre deux personnalités qu'elle se déroule : Clemenceau et Foch. Les deux hommes se disputent, département par département, la place du plus grand nombre de dénominations. 
Souvent, ces collectivités locales comptent presque autant de voies portant le nom de l'un et de l'autre. Beaucoup de villes ont à la fois une rue dénommée Clemenceau et une autre appelée Foch. Cette querelle de chiffres est d'autant plus intéressante qu'elle met en présence deux personnalités à la notoriété comparables; Clemenceau est la personnalité politique qui, par sa détermination à ne pas céder, a permis à la France de passer de l'enlisement à la décision, en 1918, tandis que Foch reste le chef de guerre, artisan de la Victoire. Clemenceau est le dirigeant politique, président du Conseil et vrai chef de l'exécutif. Foch est le chef militaire, généralissime et unificateur des commandements alliés. Deux profils, que tout opposait en 1914, se partagent, après 1918, la mémoire du conflit. Ils représentent deux camps politiques: le médecin républicain, hostile à l'Eglise catholique et le militaire de carrière conservateur et catholique pratiquant. Ils symbolisent deux camps qui cristallisent l'existence de deux France qui cohabitent dans le pays qui les a vu naître. De ce point de vue, la répartition parfois inégale s'explique par la couleur des pays qui les ont choisis, ou encore par l'origine qu'ont nos deux hommes. La France conservatrice choisit plus volontiers de mettre à l'honneur Foch, alors que les zones de forces de la France républicaine de gauche privilégient la figure de Clemenceau. On peut citer de ce point de vue la présence importante de la mémoire de celui-ci dans les départements des Bouches-duRhône, du Nord, du Pas-de-Calais.

Un autre élément joue particulièrement. Son origine permet au Tigre de dépasser son pendant dans des zones pourtant jugées réactionnaires comme la Vendée. C'est ce département qui à lui seul fait la différence, alors que la plupart des autres entités locales place les deux hommes quasiment à égalité (moins de trois de différence). D'ailleurs, la présence de l'homme qu'est Clemenceau, dans cette zone largement insurgée dès 1789 contre la Révolution, est le signe du large ralliement de la Vendée au régime républicain à l'occasion de la Grande Guerre. En 1918, cette terre reconnait l'action incontournable de celui qui l'a vu naître. Clemenceau est après la Grande Guerre, déjà un monument. Il inaugure ainsi de son vivant l'imposante statue qui lui est dédiée au carrefour de Sainte-Hermine, en Vendée (Wormser 1961, p. 441).

La mémoire de la Grande Guerre s'incarne par celle des acteurs qui y ont joué un grand rôle, ici Foch (1 307 odonymes rappellent son rôle) et Clemenceau (1 285 voies portent son nom). Or, les odonymes célébrant les personnages liés à la seconde guerre mondiale sont beaucoup plus nombreux. De Gaulle, qui est mentionné au moins autant pour son rôle de chef de l'Etat, domine les statistiques, puisque 3633 communes comptent une voie qui porte son nom, puis Leclerc réussit à être présent dans le haut des statistiques, avec les 2600 voies qui lui sont dédiées (Oulmont, 2009, p. 1-2) ${ }^{11}$.

\section{Conclusion}

L'odonymie des conflits révèle la présence des guerres dans le quotidien des Français. Le changement de position de 1918 est révélateur de celui du roman national français. Pierre Nora estime que «l'apothéose de ce roman national est la victoire de 1918, qui lui donne son sens et sa légitimité. Car la victoire confirme que la synthèse républicaine est la bonne : elle opère la fusion entre l'Etat, la nation et la République, scellée par l'union sacrée et le plus grand effort que la nation ait jamais accompli sur lui-même " (Nora, 2017, p. 13). A contrario, « les jeunes générations, nées depuis le milieu du siècle dernier, n'ont connu que des défaites : de 1940, de 1962. Par surcroît, même la victoire 
de 1918 s'est révélée dans la conscience collective comme la défaite de toute l'Europe » (Nora, 2017, p. 14).

Cependant, "les cérémonies du 11 Novembre apparaissent [...] comme le seul culte républicain qui ait réussi en France et qui ait suscité une unanimité populaire » (Prost, 1984, p. 214). C'est certainement la raison pour laquelle la loi de 2012 ne fait pas disparaître ce jour férié mais le transforme. La greffe mémorielle va-t-elle prendre ? Il faut qu'elle soit plantée sur un greffon favorable. Ainsi, la présence d'odonymes rappelant le premier conflit mondial et ses combattants constitue un «signe révélateur de mémoire » et un «facteur de mémoire » selon Robert Frank (Oulmont, 2009, p. 3). Mais elle peut subir le sort de celle de la Révolution, devenue lointaine. Ce parallèle a été déjà fait entre les deux périodes dans Présent, nation et mémoire (Nora, 2011, p. 247) dans d'autres domaines. Le dépassement de la mémoire de la Grande Guerre est un constat à faire dans bien des domaines (Becker, 2001). Les odonymes consacrés au premier conflit mondial vont-ils être oubliés ou absorbés par ceux d'autres conflits? Badariotti D., 2002. Les noms de rues en géographie. Plaidoyer pour une recherche sur les odonymes. Annales de géographie, vol. 111, nº 625.

Beaupré N., 2014. La Grande Guerre: du témoin, à l'historien, de la mémoire à l'histoire? Témoigner. Entre histoire et mémoire, $\mathrm{n}^{\circ} 118$.

Becker A., 2001. La Grande Guerre entre mémoire et oubli. In Léonard L. (dir.), La mémoire, entre histoire et politique. Paris, La documentation française.

Desbrosse X., 2014. Héros de l'Ouest, héros de l'Est: toponymie et Guerre froide de la Libération à nos jours. In Buton P., La guerre froide vue d'en bas. Paris, CNRS éditions. Gonac'h J., 2007. Pratiques de redénomination des rues à Vitrolles. In Collectif, L'acte de nommer, Une dynamique langue et discours. Paris, Presses de la Sorbonne, p. 101-104.

Hardier T., Rochas E., 2016. Pratiques et représentations des commémorations de la Grande Guerre : l'exemple des élèves du collège Eluard de Noyon (Oise). Matériaux pour l'histoire de notre temps, $\mathrm{n}^{\circ} 121-122$, p. 50-58. Largeaud J.-M., 2006. Napoléon et Waterloo : La défaite glorieuse de 1815 à nos jours. Paris, La Boutique de l'Histoire. Lemaître P., 2013. Au revoir là-haut. Paris, Albin Michel.

Millon C., 2019. Les cloches sonneront-elles la libération de la France septentrionale? In Jean- Bled J.-P., Deschodt J.-P., 1918, Demain la paix ? Paris, SPM. Milo D., 1989. Les noms de rues. In Nora P. (dir.), Les lieux de mémoire, tome II, La Nation, vol. 3. Paris Gallimard, p. 283-315. Nora P., 2011. Présent, nation et mémoire. Paris, Gallimard. Nora P., 2017. L'Histoire en France a été le nerf de l'unité nationale (Entretien par Toranian V. et Kopp R.). La Revue des Deux-mondes, novembre. Oulmont P., (dir.), 2009. Les Voies "de Gaulle» en France. Le Général dans l'espace et la mémoire des communes, Actes de la journée d'études du 12 juin 2007. Paris, Plon. Panicacci J.-L., 2001.Les lieux de mémoire toponymiques de la deuxième guerre mondiale dans les villes azuréennes. In Bouvier J.-C., Guillon J.-M., (dir.), La toponymie urbaine. Significations et enjeux. Paris, L'Harmattan.

Prost A., 1984. Les monuments aux morts. Culte républicain? Culte civique? Culte patriotique ? Pierre Nora P., Les lieux de mémoires, Paris, Gallimard. 
Rémond R., 2002. Une mémoire française. Paris, Desclée de Brouwer.

Reynié D., 2015. Mémoires à venir, une enquête sur la mémoire du XXe siècle auprès de 31172 jeunes de 16 à 29 ans, en 24 langues, dans 31 pays. Paris, Fondapol.

Todorov T., 2001. La vocation de la mémoire. In Léonard Y., La mémoire, entre histoire et politique. Paris, La documentation française.

Wormser G., 1961. La République de Clemenceau. Paris, PUF.

Zonabend F., 1980. La mémoire longue. Temps et histoire au village. Paris, PUF.

\section{NOTES}

1. Débarquement de Provence : Macron demande aux maires d'honorer les combattants africains. L'Obs, 15 août 2019.

2. Loi 2012-273 du 28 février 2012 fixant au 11 novembre la commémoration de tous les morts pour la France. JORF $n^{\circ} 0051$ du 29 février 2012, p. 3561.

3. 11 Novembre: l'hommage "à tous les morts pour la France" devant l'Assemblée mardi. Le Parisien, 8 janvier 2012.

4. Présidentielle : le 11 Novembre s'invite dans la campagne. Le Parisien, 11 novembre 2011.

5. Les grands hommes ou les grands événements se voient généralement attribuer une voie prestigieuse. Mais notre étude ne se focalise pas sur cet aspect du sujet, au demeurant pas ignoré. En effet, la hiérarchie n'est pas forcément respectée et des surprises sont constatées en la matière.

6. Nous avons choisi de recenser les appellations sous différentes formes significatives: « 11 novembre »; « 11 novembre 1918 ».

7. Nous avons écarté les références aux victoires, plus générales ou attachées comme « NotreDame des Victoires » au triomphe des armées chrétiennes à Lépante.

8. JORF du 26 octobre 1922, p. 10542.

9. En 2012, un amendement au projet de loi de commémoration nouvelle lors du 11 novembre, déposé par Jean-Claude Viollet et ses collègues de l'Assemblée, est adopté : il change la " paix » célébrée en « Paix ».

10. La ville de Lambersart, dans le Nord, compte une "rue des martyrs de la résistance ", à l'instar de celle voisine de Lomme. Si la seconde est traditionnellement de gauche, la première n'a encore jamais connu de maire de cette couleur dans l'après-guerre avec Albert Liévin (MRP), Julien Corbeil (RPF), Marcel Caloone (DVD) Jules Maillot (gaulliste).

11. Le travail de recension des odonymes portant sur les personnages s'étant illustrés pendant la seconde guerre mondiale n'est pas publié ici en annexe. Nos recherches confirment cependant les chiffres cités ici. 


\section{RÉSUMÉS}

La mémoire vivante du passé peut être mesurée par la gestion des odonymes (nom des routes et places). La présence de la Grande Guerre en France par l'étude de l'actuel fichier FANTOIR est significative. Mais le nombre d'odonymes qui commémore la seconde guerre mondiale, ou d'autres conflits, est plus important. Il faut donc s'interroger sur les causes de ce fait.

The living memory of the past can be measured by the management of odonyms (route and place names). The presence of the Great War in France by studying of the actual FANTOIR file is significant. But the number of odonyms who commemorates the second world war, or other conflicts, is more important. We have to ask what the cause of that is.

\section{INDEX}

Mots-clés : odonymie, rue, place, mémoire, ville, département, nom, guerre, municipalité Thèmes : Sur le Champ - Sur le Terrain

\section{AUTEUR}

\section{CLÉMENT MILLON}

Clément Millon, millon.clement@yahoo.fr, est chargé de conférences à l'ICES, La-Roche-sur-Yon. Il a récemment publié :

- Millon C., 2019. Les cloches sonneront-elles la libération? La libération des territoires de la France septentrionale occupée en 1918. In Actes du Colloque 1918 : Demain la paix. à paraître, p. 67-83.

- Millon C., 2018. Présence des années 1870-1871 en 1940 dans les revues universitaires de l'Allemagne nazie. In Collectif, La France en guerre, cinq années terribles. Rennes, PUR, p. 209-222. - Millon C., 2018. Les mots qualifiant le déclin français dans les écrits nazis entre 1940 et 1945. In Collectif, Le déclin dans le monde germanique, mots, discours et représentations (1914-2014). Reims, Epure, p. 147-169. 